\title{
Remarks on Graphons
}

\author{
Attila Nagy 1 \\ Department of Algebra \\ Budapest University of Technology and Economics \\ 1521 Budapest, Pf. 91, Hungary \\ e-mail: nagyat@math.bme.hu
}

\begin{abstract}
L. Lovász and B. Szegedy proved in 2006 that the limits of convergent graph sequences can be described by measurable symmetric functions $W:[0,1] \times[0,1] \rightarrow[0,1]$ called graphons. In our present paper we investigate the structure of the set of all graphons within the semigroup $\left(\mathfrak{F}\left([0,1]^{2}\right) ; \circ\right)$ of all fuzzy subsets of the unit square $[0,1]^{2}=[0,1] \times[0,1]$, where the operation $\circ$ is defined by: for every $f, g \in \mathfrak{F}\left([0,1]^{2}\right)$ and every $s \in[0,1]^{2},(f \circ g)(s)=\vee_{x \in[0,1]^{2}}(f(x) \wedge g(s))$.
\end{abstract}

Mathematics Subject Classification: 20M10; 08A72; 05C99.

Keywords: fuzzy subset, graphon, semigroup.

\section{Introduction and motivation}

Let $G_{n}$ be a sequence of finite simple graphs whose number of nodes tends to infinity. For every fixed finite simple graph $F$, let $\operatorname{hom}\left(F, G_{n}\right)$ denote the number of all homomorphisms from $F$ into $G_{n}$, that is, the edge-preserving functions from $V(F)$ into $V\left(G_{n}\right)$. Put

$$
t\left(F, G_{n}\right)=\frac{\operatorname{hom}\left(F, G_{n}\right)}{\left|V\left(G_{n}\right)\right|^{|V(F)|}} .
$$

Clearly, $t\left(F, G_{n}\right)$ is the probability that a random mapping from $V(F)$ into $V\left(G_{n}\right)$ should be a homomorphism. The sequence $G_{n}$ is called convergent if $\lim _{n \rightarrow \infty} t\left(F, G_{n}\right)$ exists for every finite simple graph $F$. Let

$$
t(F)=\lim _{n \rightarrow \infty} t\left(F, G_{n}\right) .
$$

\footnotetext{
${ }^{1}$ This work was supported by the National Research, Development and Innovation Office - NKFIH, 115288.
} 
Then $t$ is a graph parameter, that is, a function on simple graphs that is invariant under isomorphism. In [4, the authors given characterizations of graph parameters that arise in this manner; that is, the authors characterize the set $\mathfrak{T}$ of graph parameters $t$ for which there is a convergent sequence of simple graphs $G_{n}$ such that $t(F)=\lim _{n \rightarrow \infty} t\left(F, G_{n}\right)$ for every simple graph $F$. In the characterization of $\mathfrak{T}$, the symmetric and measurable functions $W$ : $[0,1]^{2}=[0,1] \times[0,1] \mapsto[0,1]$ called graphons play an important role. Recall that a function $W:[0,1]^{2} \mapsto[0,1]$ is said to be symmetric if $W(x, y)=W(y, x)$ is satisfied for all $x, y \in[0,1]$. A graph is said to be $k$-labelled ( $k$ is a positive integer) if the graph has $k$ nodes labelled by $1,2, \ldots, k$. For a $k$-labelled simple graph $F$ and a graphon $W$, the integral

$$
t(F, W)=\int_{[0,1]^{k}} \prod_{i j \in E(F)} W\left(x_{i}, x_{j}\right) d x_{1} d x_{2} \cdots d x_{k}
$$

is called the density of the graph $F$ in the graphon $W([5])$, where $E(F)$ denotes the set of all edges of $F$. In [4, Theorem 2.2] it was shown that a graph parameter $t$ belongs to $\mathfrak{T}$ if and only if there is a graphon $W$ such that $t(F)=$ $t(F, W)$ for all simple graphs $F$.

A function of a non-empty set $S$ into the real unit interval $[0,1]$ is called a fuzzy subset of $S$ (see [11]). By [3] and [7], if $*$ is an associative operation on a non-empty set $S$, then the set $\mathfrak{F}(S)$ of all fuzzy subsets of $S$ form a semigroup under the operation o defined by the following way: for arbitrary $f, g \in \mathfrak{F}(S)$ and $s \in S$,

$$
(f \circ g)(s)= \begin{cases}\vee_{s=x * y}(f(x) \wedge g(y)), & \text { if } s \in S^{2} \\ 0, & \text { otherwise }\end{cases}
$$

As every graphon is a fuzzy subset of the unit square $[0,1]^{2}$, the following problem seems interesting from a semigroup theory perspective.

Problem: If an associative operation $*$ is given on the unit square $[0,1]^{2}$, what can we say about the structure of the set $\mathcal{W}_{0}$ of all graphons in the semigroup $\left(\mathfrak{F}\left([0,1]^{2}\right) ; \circ\right)$ ? Is it true that $\mathcal{W}_{0}$ forms a substructure of $\left(\mathfrak{F}\left([0,1]^{2}\right) ; \circ\right)$ ? If so, what kind of substructure is it?

In this paper we deal with this problem in a special case: the given associative operation $*$ on $[0,1]^{2}$ satisfies the identity $(x, y) *(u, v)=(u, v)$. A semigroup $(S ; *)$ is called a right zero semigroup if it satisfies the identity $a * b=b$. With this terminology, the above problem is examined in that case when $[0,1]^{2}$ is a right zero semigroup.

We note that if $S$ is a non-empty set (and so it is a right zero semigroup), then the operation o defined in (1) has the following form:

$$
(f \circ g)(s)=\vee_{x \in S}(f(x) \wedge g(s))
$$


Throughout the paper, for a non-empty set $S,(\mathfrak{F}(S) ; \circ)$ will denote the semigroup in which the operation $\circ$ is defined by (2). Thus the purpose of this paper is to examine the structure of the set $\mathcal{W}_{0}$ of all graphons in the semigroup $\left(\mathfrak{F}\left([0,1]^{2}\right) ; \circ\right)$. Our studies consist of two parts. In Section 2 we describe the structure of the semigroup $(\mathfrak{F}(S) ; \circ)$ for an arbitrary non-empty set $S$, in Section 3 we focus on the semigroup $\left(\mathfrak{F}\left([0,1]^{2}\right) ; \circ\right)$ and its subset $\mathcal{W}_{0}$. A semigroup $S$ is called a band if every element $e$ of $S$ is an idempotent element, that is, $e^{2}=e$. A band satisfying the identity $a x a=x a$ is called a right regular band ([9]). In Section 2 we prove that if $S$ is an arbitrary nonempty set, then the semigroup $(\mathfrak{F}(S) ; 0$ ) is a right regular band (Theorem 2.6). In Section 3, applying the above result for the right regular band $\left(\mathfrak{F}\left([0,1]^{2}\right) ; \circ\right)$, we show that the set $\mathcal{W}_{0}$ of all graphons is a left ideal of $\left(\mathfrak{F}\left([0,1]^{2}\right) ; \circ\right)$. By this result, if $W$ is a graphon and $f$ is a fuzzy subset of $[0,1]^{2}$, then $f \circ W$ is a graphon. Thus, for arbitrary simple graphs $F$, we can consider the densities $t(F ; W)$ and $t(F ; f \circ W)$ of $F$ in $W$ and in $f \circ W$, respectively. In Section 3 we give an upper bound to $|t(F ; W)-t(F ; f \circ W)|$. In Theorem 3.6 we show that $|t(F ; W)-t(F ; f \circ W)| \leq|E(F)|(\sup (W)-\sup (f)) \Delta(\{W>\sup (f)\})$, where $\Delta(\{W>\sup (f)\})$ denotes the area of the set $\{W>\sup (f)\}=\{(x, y) \in$ $\left.[0,1]^{2}: W(x, y)>\sup (f)\right\}$.

For notations and notions not defined here, we refer to the paper 4 and the books [1], [6], [8], and [9].

\section{On the semigroup $(\mathfrak{F}(S) ; \circ)$, where $S$ is an arbitrary non-empty set}

For a fuzzy subset $f$ and a subset $X$ of a non-empty set $S$, let $\sup _{X}(f)=$ $\vee_{x \in X} f(x)$. Especially, let $\sup (f)=\sup _{S}(f)$. If $f$ and $g$ are arbitrary fuzzy subsets of $S$, then let $g_{f}$ and $g_{f}^{*}$ denote the following fuzzy subsets of $S$ : for an arbitrary $s \in S$, let

$$
g_{f}(s)= \begin{cases}\sup (f), & \text { if } g(s)>\sup (f) \\ g(s), & \text { otherwise }\end{cases}
$$

and

$$
g_{f}^{*}(s)= \begin{cases}g(s)-\sup (f), & \text { if } g(s)>\sup (f) \\ 0, & \text { otherwise. }\end{cases}
$$

Remark 2.1 By the above definitions, $g_{f}+g_{f}^{*}=g$ for every fuzzy subsets $f$ and $g$ of a non-empty set $S$. 
Remark 2.2 Let $f$ and $g$ be arbitrary fuzzy subsets of a non-empty set $S$. It is clear that $\sup (g) \leq \sup (f)$ implies $g(s) \leq \sup (f)$ for every $s \in S$ and so $g_{f}=g$. In case $\sup (g)>\sup (f)$, there is an element $s \in S$ such that $g(s)>\sup (f)$ and so $g_{f}(s)=\sup (f)<g(s)$. Hence $g_{f} \neq g$. Thus, for every fuzzy subsets $f$ and $g$ of $S$, the equation $g_{f}=g$ holds if and only if $\sup (g) \leq \sup (f)$.

By Remark 2.2, the following lemma holds.

Lemma 2.3 For arbitrary fuzzy subsets $f$ and $g$ of a non-empty set $S$, the equations $g_{f}=g$ and $f_{g}=f$ together hold if and only if $\sup (g)=\sup (f)$.

The next lemma will be used in Lemma 3.1

Lemma 2.4 If $f$ and $g$ are fuzzy subsets of a non-empty set $S$ such that $\sup (f) \leq \sup (g)$ then $\sup \left(g_{f}\right)=\sup (f)$ and $\sup \left(g_{f}^{*}\right)=\sup (g)-\sup (f)$.

Proof. By the definition of $g_{f}$ and $g_{f}^{*}$, it is obvious.

Theorem 2.5 Let $S$ be a non-empty set. For every fuzzy subsets $f$ and $g$ of $S$, we have $f \circ g=g_{f}$.

Proof. Let $f$ and $g$ be arbitrary fuzzy subsets of a non-empty set $S$. By the above, $(\mathfrak{F}(S) ; \circ)$ is a semigroup. Let $s$ be an arbitrary element of $S$. If $g(s)>\sup (f)$, then $f(x) \wedge g(s)=f(x)$ for every $x \in S$, and so $(f \circ g)(s)=$ $\vee_{x \in S} f(x)=\sup (f)$. If $g(s) \leq \sup (f)$, then we have two subcases.

Case 1: If $g(s)=\sup (f)$, then $f(x) \wedge g(s)=f(x)$ for all $x \in S$, and so $(f \circ g)(s)=\vee_{x \in S} f(x)=\sup (f)=g(s)$.

Case 2: If $g(s)<\sup (f)$, then there is an $x_{0} \in S$ such that $f\left(x_{0}\right)>g(s)$ and so $f\left(x_{0}\right) \wedge g(s)=g(s)$. Moreover, for arbitrary $x \in S \backslash\left\{x_{0}\right\}$, we have

$$
f(x) \wedge g(s)= \begin{cases}g(s), & \text { if } g(s)<f(x) \\ f(x), & \text { if } f(x) \leq g(s),\end{cases}
$$

and so $(f \circ g)(s)=\left(f\left(x_{0}\right) \wedge g(s)\right) \vee\left(\vee_{x \in S \backslash\left\{x_{0}\right\}}(f(x) \wedge g(s))=g(s)\right.$. Summarizing our results, we get

$$
(f \circ g)(s)= \begin{cases}\sup (f), & \text { if } g(s)>\sup (f) \\ g(s), & \text { otherwise, }\end{cases}
$$

that is, $(f \circ g)(s)=g_{f}(s)$, which proves our assertion.

A commutative band is called a semilattice. A congruence $\alpha$ on a semigroup $A$ is said to be a semilattice congruence if the factor semigroup $A / \alpha$ is a 
semilattice. A semigroup $A$ is said to be semilattice indecomposable if the universal relation is the only semilattice congruence on $A$. It is known ([10]) that every semigroup has a least semilattice congruence $\eta$; the classes of $\eta$ are semilattice indecomposable. By [9, II.3.12. Proposition], a band is a right regular band if and only if its $\eta$-classes are right zero semigroups.

Theorem 2.6 For an arbitrary non-empty set $S$, the semigroup $(\mathfrak{F}(S) ; 0)$ is a right regular band. The $\eta$-classes of $\mathfrak{F}(S)$ are right zero semigroups. Two fuzzy subsets $f$ and $g$ of $S$ are in the same $\eta$-class if and only if $\sup (f)=\sup (g)$.

Proof. Let $S$ be an arbitrary non-empty set. Then $S$ is a right zero semigroup, and so $(\mathfrak{F}(S) ; \circ)$ is a semigroup under the operation $\circ$ defined in (2), that is, $(f \circ g)(s)=\vee_{x \in S}(f(x) \wedge g(s))$ for every fuzzy subsets $f$ and $g$ of $S$ and every element $s \in S$. By Theorem 2.5, it is clear that $f \circ f=f$ for every $f \in \mathfrak{F}(S)$, and so $(\mathfrak{F}(S) ; \circ)$ is a band. Using also Theorem 2.5, we have $g \circ f \circ g=g \circ g_{f}$. As $\sup (g) \geq \sup \left(g_{f}\right)$, we have $g \circ g_{f}=g_{f}$. Thus $g \circ f \circ g=g_{f}=f \circ g$. Hence $(\mathfrak{F}(S) ; \circ)$ is a right regular band. Let $\eta$ denote the least semilattice congruence on $(\mathfrak{F}(S) ; \circ)$. The $\eta$-classes of $(\mathfrak{F}(S) ; \circ)$ are right zero semigroups by [9, II.3.12. Proposition]. Let $f$ and $g$ be arbitrary fuzzy subsets of $S$. By [9, II.1.1. Proposition], $(f, g) \in \eta$ if and only if $f \circ g \circ f=f$ and $g \circ f \circ g=g$. As $(\mathfrak{F}(S) ; \circ)$ is a right regular band, we have $f \circ g \circ f=g \circ f$ and $g \circ f \circ g=f \circ g$. Thus $(f, g) \in \eta$ if and only if $g \circ f=f$ and $f \circ g=g$. Using Theorem 2.5, $(f, g) \in \eta$ if and only if $f_{g}=f$ and $g_{f}=g$. By Lemma 2.3, we get $(f, g) \in \eta$ if and only if $\sup (f)=\sup (g)$.

\section{On the structure of the set of all graphons in the semigroup $\left(\mathfrak{F}\left([0,1]^{2}\right) ; 0\right)$}

Let $(S, \mathcal{A}, \mu)$ be a measurable space ([2]). For a fuzzy subset $h$ of $S$ and a real number $A$, let $\{h>A\}=\{s \in S: h(s)>A\}$. A fuzzy subset $h$ of $S$ is said to be measurable if, for every real number $A$, the subset $\{h>A\}$ of $S$ is measurable (that is, $\{h>A\} \in \mathcal{A}$ ).

Lemma 3.1 Let $(S, \mathcal{A}, \mu)$ be a measurable space. Then, for an arbitrary fuzzy subset $f$ and an arbitrary measurable fuzzy subset $g$ of $S$, the fuzzy subsets $g_{f}$ and $g_{f}^{*}$ are measurable.

Proof. Let $f$ and $g$ be arbitrary fuzzy subsets of $S$ such that $g$ is measurable. If $\sup (f) \geq \sup (g)$, then $g_{f}=f \circ g=g$ and $g_{f}^{*}=0$. In this case the fuzzy subsets $g_{f}$ and $g_{f}^{*}$ are measurable. Consider the case when $\sup (f)<\sup (g)$. 
Then $\sup \left(g_{f}\right)=\sup (f)$ and $\sup \left(g_{f}^{*}\right)=\sup (g)-\sup (f)$ by Lemma 2.4. Let $A$ be an arbitrary real number. It is easy to see that

$$
\left\{g_{f}>A\right\}= \begin{cases}\emptyset, & \text { if } A \geq \sup (f) \\ \{g>A\}, & \text { otherwise }\end{cases}
$$

and

$$
\left\{g_{f}^{*}>A\right\}= \begin{cases}\emptyset, & \text { if } A \geq \sup (g)-\sup (f) \\ \{g>A+\sup (f)\}, & \text { if } 0 \leq A<\sup (g)-\sup (f) \\ S, & \text { if } A<0\end{cases}
$$

from which it follows that $g_{f}$ and $g_{f}^{*}$ are measurable fuzzy subsets of $S$.

A fuzzy subset $f$ of $[0,1]^{2}$ is said to be symmetric if $f(x, y)=f(y, x)$ is satisfied for all $x, y \in[0,1]$.

Lemma 3.2 If $f$ is an arbitrary fuzzy subset and $g$ is a symmetric fuzzy subset of $[0,1]^{2}$, then $g_{f}$ and $g_{f}^{*}$ are symmetric fuzzy subsets of $[0,1]^{2}$.

Proof. It is obvious by the definition of $g_{f}$ and $g_{f}^{*}$.

Lemma 3.3 If $W$ is a graphon and $f$ is a fuzzy subset of $[0,1]^{2}$, then $W_{f}$ and $W_{f}^{*}$ are graphons.

Proof. By Lemma 3.1 and Lemma 3.2, it is obvious.

The following theorem provides an answer to the question raised in Problem in the case, where the given operation $\cdot$ on $[0,1]^{2}$ satisfies the identity $a \cdot b=b$.

Theorem 3.4 The set $\mathcal{W}_{0}$ of all graphons is a left ideal of the right regular band $\left(\mathfrak{F}\left([0,1]^{2}\right) ; \circ\right)$ of all fuzzy subsets of $[0,1]^{2}$. Thus the semigroup $\left(\mathcal{W}_{0} ; \circ\right)$ of all graphons is a right regular band, and so it is a semilattice I of right zero subsemigroups $S_{i}(i \in I)$. Two graphons $W_{1}$ and $W_{2}$ are in the same $S_{i}$ if and only if $\sup \left(W_{1}\right)=\sup \left(W_{2}\right)$.

Proof. Let $W$ be a graphon and $f$ be a fuzzy subset of $[0,1]^{2}$. By Theorem 2.5, $f \circ W=W_{f}$. Then $f \circ W$ is a graphon by Lemma 3.3. Thus the set $\mathcal{W}_{0}$ of all graphons is a left ideal of the semigroup $\left(\mathfrak{F}\left([0,1]^{2}\right) ; 0\right)$ of all fuzzy subsets of $[0,1]^{2}$. By Theorem 2.6, the semigroup $\left(\mathfrak{F}\left([0,1]^{2}\right) ; \circ\right)$ and so its subsemigroup $\left(\mathcal{W}_{0} ; \circ\right)$ is a right regular band. Moreover, the $\eta$-classes of $\mathcal{W}_{0}$ are right zero semigroups; two graphons $W_{1}$ and $W_{2}$ are in the same $\eta$-class if and only if $\sup \left(W_{1}\right)=\sup \left(W_{2}\right)$.

Let $\sigma$ denote the equivalence relation on the set $\mathcal{W}_{0}$ of all graphons defined by $\left(W_{1}, W_{2}\right) \in \sigma$ if and only if $W_{1}=W_{2}$ almost everywhere in $[0,1]^{2}$. 
Proposition 3.5 The equivalence relation $\sigma \cap \eta$ is a congruence on the right regular band $\left(\mathcal{W}_{0} ; \circ\right)$ of all graphons, where $\eta$ is the least semilattice congruence on $\left(\mathcal{W}_{0} ; \circ\right)$.

Proof. Let $W_{1}$ and $W_{2}$ be two graphons with $\left(W_{1}, W_{2}\right) \in \sigma \cap \eta$. Then, using Theorem 3.4, we have $\sup \left(W_{1}\right)=\sup \left(W_{2}\right)$ and $W_{1}=W_{2}$ almost everywhere in $[0,1]^{2}$. Let $W$ be an arbitrary graphon. As $\sup \left(W_{1}\right)=\sup \left(W_{2}\right)$, we have $W_{1} \circ W=W_{2} \circ W$. Thus $\left(W_{1} \circ W, W_{2} \circ W\right) \in \sigma \cap \eta$. Hence $\sigma \cap \eta$ is a right congruence on $\left(\mathcal{W}_{0} ; \circ\right)$. Let $T=\left\{(x, y) \in[0,1]^{2} \mid W_{1}(x, y) \neq W_{2}(x, y)\right\}$. As $\left(W_{1}, W_{2}\right) \in \sigma$, the area of $T$ is 0 . It is clear that $\left\{(x, y) \in[0,1]^{2}:(W \circ\right.$ $\left.\left.W_{1}\right)(x, y) \neq\left(W \circ W_{2}\right)(x, y)\right\} \subseteq T$ and so $\left(W \circ W_{1}, W \circ W_{2}\right) \in \sigma$. As $\left(W_{1}, W_{2}\right) \in$ $\eta$ and $\eta$ is a congruence on $\left(\mathcal{W}_{0} ; \circ\right)$, we have $\left(W \circ W_{1}, W \circ W_{2}\right) \in \eta$. Thus $\left(W \circ W_{1}, W \circ W_{2}\right) \in \sigma \cap \eta$ and so $\sigma \cap \eta$ is a left congruence on $\left(\mathcal{W}_{0} ; \circ\right)$. Thus $\sigma \cap \eta$ is a congruence on $\left(\mathcal{W}_{0} ; \circ\right)$.

Let $W$ be a graphon and $f$ a fuzzy subset of $[0,1]^{2}$. By Theorem 3.4 , $f \circ W$ is a graphon. Thus, for arbitrary simple graphs $F$, we can consider the densities $t(F ; W)$ and $t(F ; f \circ W)$ of $F$ in $W$ and $f \circ W$, respectively. The next theorem gives an upper bound to $|t(F ; W)-t(F ; f \circ W)|$.

Theorem 3.6 Let $W$ be an arbitrary graphon. Then, for an arbitrary fuzzy subset $f$ of $[0,1]^{2}$ and an arbitrary finite simple graph $F$,

$$
|t(F ; W)-t(F ; f \circ W)| \leq|E(F)|(\sup (W)-\sup (f)) \Delta(\{W>\sup (f)\}),
$$

where $E(F)$ denotes the set of all edges of $F$ and $\Delta(\{W>\sup (f)\})$ denotes the area of the set $\{W>\sup (f)\}=\left\{(x, y) \in[0,1]^{2}: W(x, y)>\sup (f)\right\}$.

Proof. Let $W$ be an arbitrary graphon and $f$ an arbitrary fuzzy subset of $[0,1]^{2}$. By Theorem [3.4, $f \circ W$ is a graphon. If $\sup (W) \leq \sup (f)$, then $W=f \circ W$ and $\{W>\sup (f)\}=\emptyset$. Thus $|t(F ; W)-t(F ; f \circ W)|=$ $0=|E(F)|(\sup (W)-\sup (f)) \Delta(\{W>\sup (f)\})$. Consider the case when $\sup (W)>\sup (f)$. By Remark 2.1, $W-(f \circ W)=W_{f}^{*}$. As $W$ is a graphon, $W_{f}=f \circ W$ and $W_{f}^{*}$ are graphons by Lemma 3.3. Thus $W, f \circ W$ and $W_{f}^{*}$ are integrable functions on $[0,1]^{2}$. Using [4, Lemma 4.1], $|t(F ; W)-t(F ; f \circ W)| \leq$ $|E(F)| \cdot\left\|W_{f}^{*}\right\|_{0}$, where $\left\|W_{f}^{*}\right\|_{0}=\sup _{\substack{A \subseteq[0,1] \\ B \subseteq[0,1]}}\left|\int_{A} \int_{B} W_{f}^{*}(x, y) d x d y\right|$. As $W_{f}^{*}$ is a non-negative function, $\left\|W_{f}^{*}\right\|_{0}=\left\|W_{f}^{*}\right\|_{1}$, where $\left\|W_{f}^{*}\right\|_{1}=\int_{0}^{1} \int_{0}^{1}\left|W_{f}^{*}(x, y)\right| d x d y$. Thus $|t(F ; W)-t(F ; f \circ W)| \leq|E(F)| \cdot|| W_{f}^{*} \|_{1}$. As $W_{f}^{*}(x, y)=0$ for all $(x, y) \in[0,1]^{2} \backslash\{W>\sup (f)\}$, we have $\left\|W_{f}^{*}\right\|_{1}=\int_{0}^{1} \int_{0}^{1} W_{f}^{*}(x, y) d x d y \leq$ $(\sup (W)-\sup (f)) \Delta\left(\{W>\sup (f)\}\right.$, because $\sup \left(W_{f}^{*}\right)=\sup (W)-\sup (f)$ by Lemma 2.4. Consequently $|t(F ; W)-t(F ; f \circ W)| \leq|E(F)|(\sup (W)-$ $\sup (f)) \Delta(\{W>\sup (f)\})$. 


\section{References}

[1] A.H. Clifford and G.B. Preston, The Algebraic Theory of Semigroups I., Amer. Math. Soc. Providence R.I., 1961.

[2] D.L. Cohn, Measure Theory, Birkhäuser Boston, Inc., Boston, MA, 1993.

[3] W. Liu, Fuzzy Invariant Subgroups and Fuzzy Ideals, Fuzzy Sets and Systems, 8(1982), pp. 133-139.

[4] L. Lovász and B. Szegedy, Limits of dense graph sequences, Journal of Combinatorial Theory, Series B, 96(2006), pp. 933-957.

[5] L. Lovász and B. Szegedy, Random Graphons and a Weak Positivstellensatz for Graphs, Journal of Graph Theory, 70:2 (2012), pp. 214-225.

[6] L. Lovász, Large networks and graph limits, American Mathematical Society, Rhode Island, 2012.

[7] J.N. Mordeson, D.S. Malik and N. Kuroki, Fuzzy Semigroups, Springer, Berlin, 2003.

[8] A. Nagy, Special Classes of Semigroups, Kluwer Academic Publishers, Dordrecht/Boston/London, 2001.

[9] M. Petrich, Lectures in Semigroups, Akademie-Verlag Berlin, 1977.

[10] T. Tamura, Note on the greatest semilattice decomposition of semigroups, Semigroup Forum, 4(1972), pp. 255 - 261.

[11] L.A. Zadeh. Fuzzy Sets, Information and Control, 8(1965), pp. 338-353. 